\title{
Nanotechnology in Dentistry- A Review
}

\author{
Saranya George ${ }^{1}$ \\ ${ }^{I}$ (Oral Medicine And Radiology, Government Dental College Trivandrum, Kerala University Of Health \\ Sciences, India)
}

\begin{abstract}
Nanotechnology is manipulating matter at nanometre level. Applications of nanotechnology for treatment, diagnosis, monitoring, and control of biological systems has recently been referred to as "nanomedicine" by the National Institutes of Health. It holds promise for advanced diagnostics, targeted drug delivery and biosensors. In the long term, medical nanorobots will allow instant pathogen detection and elimination as well as individual cell surgery in vivo. Dentistry is in no way exempted from this revolutionary technical advance in science.
\end{abstract}

Keywords: Nanodentistry, nanomedicine, nanorobots

\section{Introduction}

The word "nano," is derived from the Greek word (nannos) meaning "dwarf," which literally refers to 1 billionth of a physical size. ${ }^{1}$ One nanometer $(\mathrm{nm})$ is a unit of length that equals 1 billionth of a meter. The size of atoms is approximately $0.1 \mathrm{~nm}$. The size of a usable nanostructure is 1 to $100 \mathrm{~nm}$. It means the area of nanotechnology works at the level of atoms and molecules. ${ }^{1}$ The material properties have significantly altered following the micro-to-nano shift in the scale. Hence a new field was born to explain these rather strange phenomena, named nanoscience and the application of its discoveries termed nanotechnology. ${ }^{2}$

Disease diagnosis, treatment, and prevention. These technological innovations, referred to as nanomedicines by the National Institutes of Health (Bethesda, MD, USA), have the potential to turn molecular discoveries arising from genomics and proteomics into widespread benefit for patients. Nanomedicine is a large subject area and includes nanoparticles that act as biological mimetics (e.g., functionalized carbon nanotubes), "nanomachines" (e.g., those made from interchangeable DNA parts and DNA scaffolds such as octahedron and stick cube), nanofibers and polymeric nanoconstructs as biomaterials (e.g., molecular self-assembly and nanofibers of peptides and peptide-amphiphiles for tissue engineering, shape-memory polymers as molecular switches, nanoporous membranes), and nanoscale microfabrication-based devices (e.g., silicon microchips for drug release and micromachined hollow needles and two-dimensional needle arrays from single crystal silicon), sensors and laboratory diagnostics. New potential treatment opportunities in dentistry include, local anesthesia, dentition renaturalization, permanent hypersensitivity cure, complete orthodontic realignments during a single office visit, covalently bonded diamondised enamel, and continuous oral health maintenance using mechanical dentifrobots. ${ }^{3}$

\section{History Of Nanotechnology}

Nanoparticles were used by artisans as far back as the 9th century in Mesopotamia for generating a glittering effect on the surface of pots. ${ }^{3}$ Pottery from the Middle Ages and Renaissance often retain a distinct gold or copper colored metallic luster. ${ }^{3}$ The luster originated within the film itself, which contained silver and copper nanoparticles dispersed homogeneously in the glassy matrix of the ceramic glaze. ${ }^{4}$

The concepts that seeded nanotechnology were first discussed in 1959 by renowned physicist Richard Feynman in his talk "There's Plenty of Room at the Bottom" in which he described the possibility of synthesis via direct manipulation of atoms. The term "nano-technology" was first used by Norio Taniguchi in 1974, though it was not widely known. ${ }^{2,3}$

In the 1980s, two inventions which enabled the imaging of individual atoms or molecules as well as their manipulation led to significant progress in the field of nanotechnology. Gerd Binnig invented scanning tunnelling microscopy (STM) while Henrich Rohrer invented atomic force microscopy. Inspired by Feynman's concepts, K. Eric Drexler independently used the term "nanotechnology" in his 1986 book Engines of Creation: The Coming Era of Nanotechnology.

In 1991, Saumio Iijima discovered carbon nanotubes and by 2000, the United States government launched the National Nanotechnology Initiative (NNI - a Federal visionary research and development programme for nanotechnology-based investments through the coordination of 16 various US departments and independent agencies) and these paved way for the progress in research and development in the field of nanotechnology. ${ }^{4}$ 


\section{Synthesis Of Nanoparticles:}

There are two main approaches for the synthesis of nano-engineered materials. They can be classified on the basis of how molecules are assembled to achieve the desired product ${ }^{5}$.

\subsection{Top - down technique}

The top - down technique begins with taking a macroscopic material (the finished product) and then incorporating smaller scale details into them. The molecules are rearranged to get the desired property. This approach is still not viable as many of the devices used to operate at nano level are still being developed. ${ }^{5,6,7}$

\subsection{Bottom - up approach}

The bottom - up approach begins by designing and synthesizing custom made molecules that have the ability to self- replicate. These molecules are then organized into higher macro-scale structures. The molecules self-replicate upon the change in specific physical or chemical property that triggers the self-replication. This can be a change in temperature, pressure, application of electricity or a chemical. The self-replication of molecule has to be carefully controlled so it does not go out of hand. ${ }^{5,6,7}$

\section{Nanodentistry}

The concept of nanomedicine was first put forward in 1993 by Robert A. Freitas Jr. and was defined as observing, controlling, and treating the biological systems of the human body at the molecular level using nanostructures and nano-devices ${ }^{7}$. . New potential treatment opportunities in dentistry may include: local anaesthesia, dentition renaturalization, and permanent hypersensitivity cure, visit, covalently bonded diamondised enamel, and continuous oral health maintenance using mechanical dentifrobots. ${ }^{12}$

\subsection{Nanotechnology in Oral surgery \\ 4.1.1 Orofacial fain management}

Dental nanorobots use specific motility mechanisms to crawl or swim through human tissue with navigational precision, acquire energy, sense, and manipulate their surroundings, achieve safety to penetration and use any of the multitude techniques to monitor, interrupt, or alter nerve impulse traffic in individual nerve cells in real time. ${ }^{10}$ These nanorobot functions may be controlled by an on board nanocomputer that executes pre-programmed instructions in response to local sensor stimuli. Alternatively, the dentist may issue strategic instructions by transmitting orders directly to in vivo nanorobots via acoustic signals or other means they can specifically useful in treating MPDS, Trigeminal Neuralgias, early cancer detection. ${ }^{10,12}$

\subsubsection{Local nanoanaesthesia:}

A colloidal suspension containing millions of active analgesic micron-size dental robots will be instilled on the patient's gingiva. After contacting the surface of crown or mucosa, the ambulating nanorobots reach the pulp via the gingival sulcus, lamina propria and dentinal tubules. Once installed in the pulp, the analgesic dental robots may be commanded by the dentist to shut down all sensitivity in any particular tooth that requires treatment. After oral procedures are completed, the dentist orders the nanorobots to restore all sensation, to relinquish control of nerve traffic and to egress from the tooth by similar pathways used for ingress $^{12}$

\subsubsection{Nanoencapsulation}

South West Research Institute (SWRI) has developed targeted release systems that encompass nanocapsules including novel vaccines, antibiotics, and drug delivery with reduced side effects. ${ }^{12}$

\subsubsection{Nanoneedles}

Suture needles incorporating nano-sized stainless steel crystals have been developed (trade name: Sandvik Bioline, RK 91 needles, AB Sandvik, Sweden). Nano tweezers are also under development, which will make cell surgery possible in the near future. ${ }^{13}$

\subsubsection{Bone Replacement Materials:}

Bone is a natural nanostructured (ie, a material with constituent features less than $100 \mathrm{~nm}$ in at least one dimension) composite composed of organic compounds (mainly collagen) reinforced with inorganic ones. It is this natural nanostructure that nanotechnology aims to emulate for orthopedic and dental applications. The nanocrystallites show a loose microstructure, with nanopores situated between the crystallites. This material structure will be completed by pores in the micrometer area. By following this process, a rough surface area is formed on the boundary layer between the biomaterial and cell, which is very important for fast cell growth. Special features of bone graft materials are as follows: $:^{12,13}$ 
- Osteoinductive

- Fully synthetic

- Not sintered

- Highly porous

- Nanostructured

- Absorbs natural body-produced proteins into the nanopores

- Degradation by osteoclasts

- Very good processibility

- No ionic solution products

\subsection{Nanotechnology in Prosthodontics:}

\subsubsection{Impression materials:}

Nanofillers are integrated in vinyl polysiloxanes, producing a unique addition of siloxane impression materials. The material has better flow, improved hydrophilic properties and enhanced detail precision. ${ }^{14}$

\subsubsection{Implants:}

Understanding and controlling interfacial reaction at nano level has led to production of implants with nanoscale topography and coatings for better and faster osseointegration designed to function a lifetime of the individual. ${ }^{13}$ Drug delivery provide local dose of anti-inflammatory agents that is gradually released from coatings on the surface of implanted device.

\subsection{Nanotechnology in Orthodontics:}

Orthodontic nano-robots could directly manipulate the periodontal tissues, allowing rapid and painless tooth straightening, rotating and vertical repositioning within minutes to hours. Optiflex impregnated with nano composite materials is revolutionary in orthodontics. With nanotechnology orthodontic realignments can be completed in a single office visit. ${ }^{14}$

\subsection{Nanotechnology in Periodontics: \\ 4.4.1 Dentinal hypersensitivity:}

Reconstructive dental nanorobots, using native biological materials, could selectively and precisely occlude specific tubules within minutes, offering patients a quick and permanent cure. On reaching the dentin, the nanorobots enter dentinal tubular holes and proceed toward the pulp, guided by a combination of chemical gradients, temperature differentials and even position of navigation, all under the control of the on-board nano computer as directed by the dentist. When the dentist presses the icon for the desired tooth on the hand held controlled display monitor, it is immediately anesthetized. ${ }^{15}$

\subsubsection{Nanomaterials for periodontal drug delivery:}

An effective and satisfactory drug delivery system for the treatment of periodontal diseases has been developed by producing nanoparticles impregnated with triclosan

\subsubsection{Dentifrobots:}

Nanorobotic dentifrice (dentifrobots) delivered by mouthwash or toothpaste could patrol all supragingival and subgingival surfaces at least once a day metabolizing trapped organic matter into harmless and odourless vapours and performing continuous calculus debridement. ${ }^{15}$

\subsubsection{Tissue Engineering And Dentistry:}

Potential applications of tissue engineering and stem cell research in dentistry include the treatment of orofacial fractures, bone augmentation, cartilage regeneration of the temporomandibular joint, pulp repair, periodontal ligament regeneration, and implant osseointegration.

\subsection{Nanotechnology in Conservative dentistry: \\ 4.5.1 Nanocomposites:}

Nanotechnology has enabled the production of nano-dimensional filler particles, which are added either singly or as nanoclusters into composite resins. Nanotechnology allows the production of nano-sized filler particles can be added in greater amounts into the composite resin matrix unlike traditional fillers. ${ }^{17}$ These composites produce a smooth surface after the polishing process and confer superior esthetic features to the material.

The fillers in nano- composites have higher translucence since they are smaller than the wavelength of light, allowing the generation of more esthetic restorations with a vast range of color options. 


\subsubsection{Nanosolution}

Nanosolutions produce unique and dispersible nanoparticles, which can be used in bonding. agents (trade name: Adper Single Bond Plus Adhesive Single Bond). ${ }^{16}$

\subsubsection{Nanocomposite Artificial Teeth}

Artificial teeth made of nanocomposite have also been produced. In these artificial teeth, inorganic fillers in nano-dimensions are diffused homogenously without any accumulation in the matrix. Therefore, the smoothness of the surface can be preserved even when the teeth are eroded. Tests have shown that nanocomposite artificial teeth are more durable than acrylic teeth and microfill composite teeth and have a higher resistance to abrasion. Moreover, composite resin artificial teeth containing nanofiller show superior color. ${ }^{16}$

\subsubsection{Tooth Repair}

Nanorobotic manufacture and installation of a biologically autologous whole replacement tooth that includes both mineral and cellular components (ie, complete dentition replacement therapy) should become feasible within the time and economic constraints of a typical office visit through the use of an affordable desktop manufacturing facility. ${ }^{16}$

\subsubsection{Tooth Renaturalization}

This technology enables the affected teeth remanufactured to become indistinguishable from original teeth. ${ }^{17}$ Dentin bonding agents using nanotechnology bond with dentin and composite restorative material forming a firm bond. They have superior properties compared to the conventional ones.

\subsubsection{Nanotechnology For Prevention Of Dental Caries:}

The use of a toothpaste containing nanosized calcium carbonate enabled remineralization of early enamel lesions.

\subsubsection{Tooth Durability And Appearance:}

Durability and appearance of tooth may be improved by replacing upper enamel layers with covalently bonded artificial materials such as sapphire or diamond, which have 20-100 times the hardness and failure strength of natural enamel or contemporary ceramic veneers and good biocompatibility. Pure sapphire and diamond are brittle and prone to fracture, can be made more fracture resistant as part of a nanostructured composite material that possibly includes embedded carbon nanotubes. ${ }^{17}$

\subsection{Nanotechnology in Oral medicine and radiology: \\ 4.6.1 Nanodiagnostics:}

Nano diagnostics is the use of nano devices for the early disease identification. In in-vitro diagnostics, nano medicine could increase the efficiency and reliability of the diagnostics using human fluids saliva or tissues samples by using selective nano devices, to make multiple analyses at sub cellular scale. In in- vivo diagnostics, nano medicine could develop devices able to work inside the human body in order to identify the early presence of a disease, to identify and quantify toxic molecules, tumor cells. ${ }^{18}$

\subsubsection{Nanorobots:}

Nanorobots may release inhibitors, antagonists or down regulators for the pyrogenic pathway in a targeted fashion to selectively absorb the endogenous pyrogens, chemically modify them, and then release them back into the body in a harmless inactivated form. The non-pyrogenic nanorobots used in vivo are bulk teflon, carbon powder and monocrystal sapphire. Pyrogenic nanorobots are alumina, silica and trace elements like copper and zinc.

\subsubsection{Diagnosis And Imaging:}

Scientists have successfully produced microchips that are coated with human molecules. The chip is designed to emit an electrical impulse signal when the molecules detect signs of a disease. Special sensor nanobots can be inserted into the blood under the skin where they check blood contents and warn of any possible diseases. They can also be used to monitor the sugar level in the blood. Advantages of using such nanobots are that they are very cheap to produce and easily portable. ${ }^{18}$ 


\subsubsection{Diagnosis And Management Of Oral Cancer: \\ Nanoscale cantilevers}

These are flexible beams resembling a row of diving boards that can be engineered to bind to molecules associated with cancer ${ }^{20}$.

Nanopores

These are tiny holes that allow DNA to pass through one strand at a time. They will make DNA sequencing more efficient. ${ }^{20}$

\section{Nanotubes}

These are carbon rods about half the diameter of a molecule of DNA that not only can detect the presence of altered genes but also may help researchers pinpoint the exact location of those changes. ${ }^{20}$

\section{Quantum dots}

Quantum dots are nanomaterials that glow very brightly when illuminated by ultraviolet light. They can be coated with a material that makes the dots attach specifically to the molecule to be tracked. Quantum dots bind themselves to proteins unique to cancer cells, literally bringing tumors to light. ${ }^{19,20}$

\section{Dendrimers}

These are highly branched macromolecules with a controlled three-dimensional architecture. The branched structure makes it possible to attach other molecules like drugs and contrast agents to the cancer cell surface. ${ }^{20}$

\section{Nanoshells}

These are miniscule beads coated with gold. By manipulating the thickness of the layers making up the nanoshells, scientists can design these beads to absorb near-infrared light, creating an intense heat that is lethal to cancer cells. Nanoshells have a core of silica and a metallic outer layer.

\section{Nanotechnology in cancer pain:}

Nanotechnology has exhibited a remarkable progress over the past 20 years in the management of pain in cancer patients. Recent applications at the nanoscale level include novel drug-delivery systems, such implantable drugdelivery devices, transdermal or transmucosal patches, and micro-needles. Oral transmucosal fentanyl citrate (OTFC; Actiq ${ }^{\circledR}$, Cephalon, UK) is the first medication developed specifically for the treatment of breakthrough pain and provides its active ingredient, fentanyl, in a unique oral transmucosal delivery system, utilizing microfabrication technology, offering personal pain control for cancer patients.

\subsubsection{Digital Dental Imaging:}

In digital radiographies obtained by nanophosphor scintillators, the radiation dose is diminished and high quality images are obtained.

\section{Conclusion}

Nanotechnology is still in its early stages. The applications discussed here have already been developed and are already helping patients all over the world. As further research continues in this field, more treatments will be discovered. Many diseases that do not have cures today may be cured by nanotechnology in the future. Scientists who are against the use of nanotechnology also agree that advancement in nanotechnology should continue because this field promises great benefits, but testing should be carried out to ensure the safety of the people. Nanotechnology will one day become part of our everyday life and will help save many lives.

\section{References}

[1]. Saravana Kumar R, Vijayalakshmi R: Nanotechnology in Dentistry, Ind J Den Res 17(2):62-65, 2006

[2]. Sule Tugba Ozak, Pelin Ozkan:Nanaotechnology and Dentistry .Eur J Dent 2013;7:145-151

[3]. Nelson et al : Nanotechnology and Drug Delivery Part 1: Background and Applications , Tropical Journal of Pharmaceutical Research, June 2009; 8 (3): 265-274

[4]. Mark T. Swihart : Vapor-phase synthesis of nanoparticles, Current Opinion in Colloid and Interface Science 8 (2003) 127-133

[5]. Sneha, Shivkumar: The Nano era in Dentistry; Journal of Natural science,Biology and Medicine, January 2013 Vol 4,issue 1

[6]. Rosaiah Kanaparthy,Aruna Kanaparthy:The changing Face of Dentistry: Nanotechnology, Int J Nanomedicine 2011;6:2799-2804

[7]. S. Moein Moghimi,1 A. Christy Hunter,and J. Clifford Murray Nanomedicine: current status and future prospects . The FASEB Journal Vol. 19 March 2005

[8]. Jeffery Jameson :Nanotechnology: Applications in medicine and possible side- effects.

[9]. K V Prabhakar Rao et al :nanotechnology in Dentistry, KDJ Vol -36, January 2013 51-54

[10]. Sujatha et al :Naorobotics -a futuristic approach:SRM University journal of dental sciences, volume1-issue1-june 2010

[11]. Sandrine Lavenus, Guy Louarn,and Pierre Layrolle Review Article Nanotechnology and Dental Implants: International Journal of Biomaterials Volume 2010, Article ID 91532

[12]. Vuk Uskoković and Luiz Eduardo Bertassoni : Review Nanotechnology in Dental Sciences: Moving towards a Finer Way of Doing Dentistry Materials 2010,3, 1674-1691

[13]. J bhuvaneswarri et al : Future impact of nanotechnology in dentistry - a review. International Journal of Nanotechnology and Application (IJNA) ISSN 2277-4777 Vol. 3, Issue 2, Jun 2013, 15-20

[14]. Rita et al :Nanodentistry: Exploring the beauty of miniature; J Clin Exp Dent.2012;4(2)

[15]. Chandra Mouli etal: Nanotechnology in dentistry- A review; Int J Bio Med Res. 2012:1550-1553

[16]. Mallanagouda Patil etal: Future impact of nanotechnology on medicine and dentistry:Journal of Indian Society of PeriodontologyVol12,issue2,May-Aug 2008 
[17]. Anvitha Sharath Kumar and Lavanya R Nanotechnology: A Changing Face in Modern Era :RRJDS Volume 1 , Issue 2 July September, 2013

[18]. Suresh et al :Nanotechnology :The emerging science in dentistry; Journal of Orofacial Research Jan-March 2012;2(1) 33-36

[19]. Sanjeev Natl et al: A critical review of the implication of nanotechnology in modern dental practice .J Maxillofac Surg. 2010 JanJun; 1(1): 41-44

[20]. Mujoo et al :Nanotechnology : A new era in dentistry. INDIAN journal of dental research and review apr 2012 - sept 2012 